\title{
Forsting M, Wanke I (Eds): Intracranial vascular malformations and aneurysms
}

\author{
Springer Verlag, Berlin, Heidelberg, 2008. ISBN 978-3-540-32920-6
}

\author{
Ulrich Sure • Erol I. Sandalcioglu
}

Received: 12 March 2009 / Accepted: 20 March 2009/Published online: 4 April 2009

(C) Springer-Verlag 2009

This publication is a continuation of the "Encyclopedia of Medical Radiology" edited by Baert, Knauth, and Sartor. This book is primarily directed to neuroradiologically interested colleagues and consists of five nicely illustrated chapters dealing with intracranial vascular malformations and aneurysms. These chapters each describe pathology, clinical presentation, imaging, and therapy of the various lesions.

The first chapter nicely illustrates and describes the various phenotypes of developmental venous anomalies (DVA). It is correctly stated that such lesions usually do not carry a risk for hemorrhage and thus should not even be followed up with the exception of an associated cavernoma that might need surgical excision. The second chapter deals with cavernomas and capillary teleangiectasias. The illustrations are of a high quality and comprise computed tomography (CT), magnetic resonance (MR; $1.5-7 \mathrm{~T}$ ), intraoperative, and angiographic images. Although a very nice summary of the literature upon cavernomas, we do not agree with the authors that some clinically progressive cavernomas with deep brain localization should be referred to radiosurgical treatment. The third chapter describes pial arteriovenous malformations (AVMs). The pathology is eloquently described and nicely illustrated. The surgical, interventional, and radiosurgical treatment options are described. A strong bias is set on the complete and incomplete interventional occlusion of AVMs with special consideration of embolization techniques and material, including onyx. The problem of flow-associated aneurysms

U. Sure $(\bowtie) \cdot$ E. I. Sandalcioglu

Klinik für Neurochirurgie, Universitätsklinikum Essen,

Essen, Germany

e-mail: ulrich.sure@uni-due.de is also discussed and well-illustrated. A recommended therapeutic strategy is well-summarized. The next chapter comprises the topic dural AVMs both of the cranial and the spinal compartments. The various types of fistulas are very well-described and illustrated in CT, MR, intraoperative, and angiographic images. A number of highly useful artistic sketches also support this nice chapter that clearly states that an endovascular approach sometimes fails and requires complex neurosurgical procedures. The last chapter describes the interventional therapy of aneurysms. The whole variety of aneurysm localization and pathology is shown. A number of sometimes highly complex and spectacular cases are illustrated in a remarkable image quality. The final remarks of this section state that $80 \%$ of aneurysms can be treated by modern endovascular techniques. Even with modern stenting and coiling techniques, these numbers are definitely too high in our recent experience; however, the "remaining" aneurysms are usually highly complex and require a specialized neurosurgical expertise that is difficult to maintain (and even more, to train) with low numbers of surgical clipping procedures in a neurovascular center.

Overall, the present book contains a very nice summary of neurosurgically relevant neurovascular topics and highly recommendable illustrations. It was edited and written by interventionally active colleagues with a bias on their primary interest. Nonetheless, we recommend keeping this book in the book shelf for any neurovascular neurosurgeon in order to furthermore support the interdisciplinary treatment strategies that are definitely of advantage for our patients.

Ulrich Sure

Erol I. Sandalcioglu

Essen, Germany 